ESAIM: PROCEEDINGS, November 2002, Vol.12, 1-7

M.Thiriet, Editor

\title{
FROM MEDICAL IMAGES TO COMPUTATIONAL MESHES
}

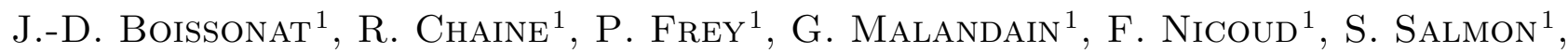 \\ E. SAltel ${ }^{1}$ And M. Thiriet ${ }^{1}$
}

\begin{abstract}
Numerical simulations take a bigger importance in medical activity as they allow to obtain information non-invasively. To take into account individual variability, we propose numerical simulations in geometries reconstructed from medical images. We present the necessary treatment of images of diseased vessels, to provide a computational mesh of an anatomical model of the subject. First a faceted surface is extracted from the images. Then this surface is transformed into a geometrical model to be finally remeshed for a finite element use.
\end{abstract}

Résumé. Les simulations numériques ayant trait au biomédical prennent de plus en plus d'importance car elles permettent d'obtenir de nouvelles informations impossibles à mesurer de manière non-invasive chez les patients. Pour prendre en compte la variabilité inter-sujet, nous proposons des simulations numériques dans des géométries reconstruites à partir de l'imagerie médicale. Dans la suite, nous présentons le traitement d'images de vaisseaux malades, nécessaire à l'obtention d'un maillage de calcul du modèle anatomique du patient. En premier lieu, on extrait des images un ensemble de facettes représentant une surface. Puis cette surface est transformée en un modèle géométrique qui est finalement remaillé pour satisfaire les contraintes d'un maillage éléments finis.

\section{INTRODUCTION}

Large-artery diseases as aneurisms or stenoses are a major cause of death in western societies. Mini-invasive treatments are associated with catheter-based procedures for implantation of medical devices in the diseased vascular region. Since haemodynamical factors contribute to the prognosis, numerical tests of blood flow behavior during a whole cardiac beat in geometrical model of the vascular segment of interest provide the informations for treatment planning and ensure better therapy. Three-dimensional reconstruction of the in vivo patient anatomy, characterized by a huge between-subject variability and, consequently, by flow differences, from medical imaging is the first step of the numerical simulations.

The present work is carried out by an interdisciplinary group which includes a set of INRIA teams through a Cooperative Research Initiative VITESV (Tab. 1). The joint investigation is aimed at developing simulators for interventional medicine based on numerical simulations of blood flows in diseased vessels meshed after three-dimensional reconstruction from medical imaging. The simulator is aimed, in particular, at providing a flexible computational support to plane the operation and at predicting complication risks by computing both blood and vascular-wall stresses. Besides, another goal is optimization of endovascular implanted devices. The present communication deals with the 3D reconstruction from medical images and the derived computational mesh. Two different saccular aneurisms, an accidental edge - and a congenital branching lesions, illustrate the

${ }^{1}$ INRIA, ARC VITESV, B.P.105, 78153 Le Chesnay Cedex, FRANCE

(c) EDP Sciences, SMAI 2003 


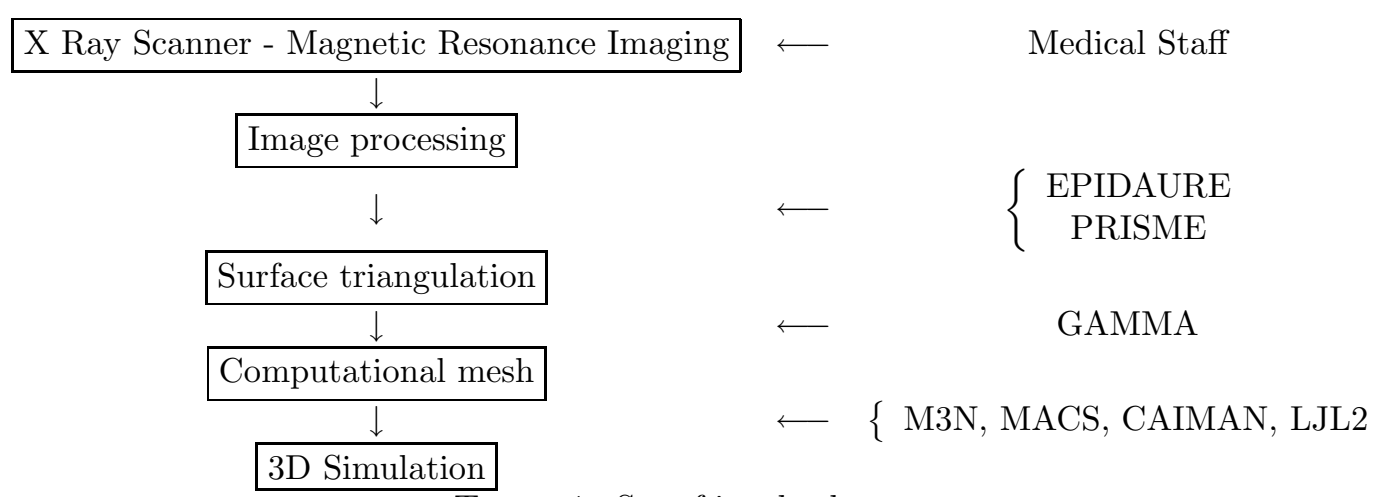

TABLE 1. Set of involved teams.

sequence of operations used to compute the blood flow in the diseased vascular region which will be treated by coils and stent when the branch is compressed by the wall dilation. The first section is devoted to the surface triangulation from the medical images. Section 2 gives the method which provides the computational meshes from the surface triangulation.

\section{IMAGE PROCESSING}

\subsection{Congenital cerebral aneurism}

The congenital terminal aneurism of a branch of the middle cerebral artery is reconstructed from X-ray scan (Fig. 1). The saccular biloculated short-necked aneurism is located at the apex of the bifurcation.

For the first image (see Figure 1), the Epidaure team in Inria Sophia uses two methods to extract vessels
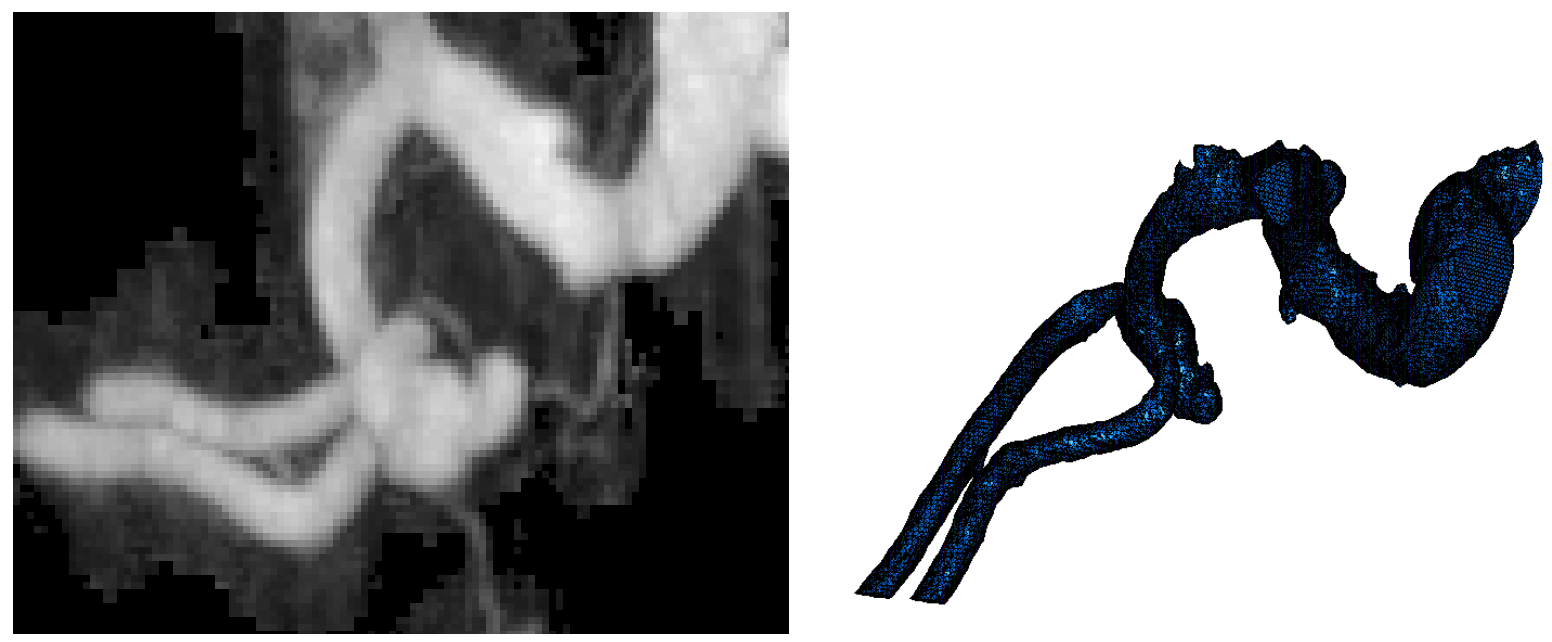

Figure 1. The congenital cerebral aneurism - RX scanner (left) and the total mesh reconstructed by EPIDAURE (right).

from the images. The first one is based on a visualization technique of 3D iso-surfaces [3]. Some artefacts are generated as there is no significant threshold. The second method is associated to a vascular modeling with the 
following assumptions. The vessel cross section, supposed to be circular, provides an image signal which fits a Gaussian intensity profile. The first and the second derivatives of the intensity signal is maximal at the wall and at the vessel axis respectively. When the axis is determined and the local radii are estimated, the vessel wall is reconstructed. Such a technique allows not only to compute transversal geometrical data, i.e. data of the vessels cross sections which have to be known for the exploitation of numerical computations, but also to extract a vessel network almost topologically perfect. But, in the case of great local variations of the diameter of the vessel, this technique leads to an artificial smoothing.

\subsection{Accidental iliac aneurism}

The second image obtained by Rangueil hospital in Toulouse shows a probably accidental aneurism, at the edge of the common iliac artery, slightly upstream from the bifurcation between the internal and the external iliac arteries. The neck is broad and high.

The artery contours correspond to the scan slices, with a between-slice distance of three millimeters (Fig. 2).
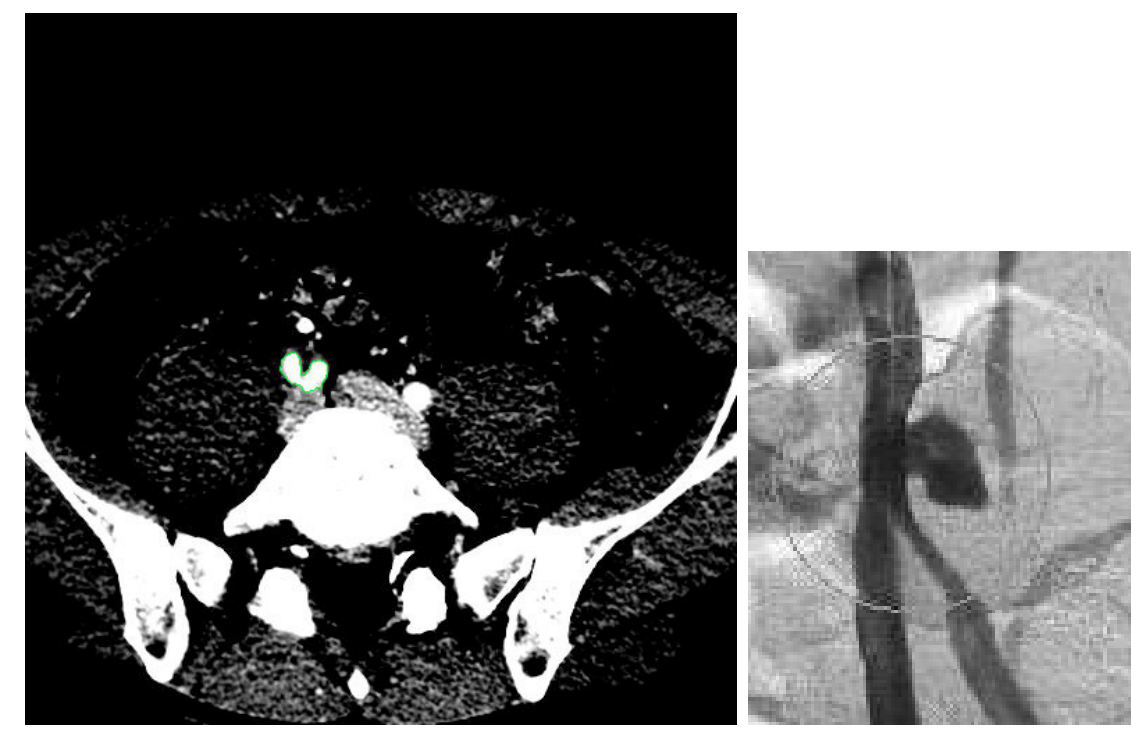

Figure 2. An abdominal slice - CT Scanner after injection of a contrasting product. The artery with the aneurism is marked in green (left) - The a posteriori angiography (right).

These contours were obtained directly from the medical images by a contour detection algorithm (Fig. 3) [5]. From these contours, the surface is reconstructed first "by hand" in Toulouse (Model I) [5], then either by both PRISME and GAMMA tools (Model II) [7] or by GAMMA softs (Model III) [6].

Model I. is composed of the common iliac artery with its edge aneurism and its bifurcation. The geometrical model is approximated since the saved slices are spaced out too far from each other (see Fig. 3 ) but is a posteriori validated by an additional angiography (see Fig. 2).

Model II. has been obtained from NUAGE, a software dedicated to surface reconstruction from contours. This software has been developed in the Prisme team at Inria Sophia Antipolis. A triangulated surface is drawn inbetween each pair of consecutive cross-sections, ensuring that each point in a contour is connected to its closest point in the next contour. The algorithm proceeds as follows : the set of points located in each $2 \mathrm{D}$ cross-section $\mathrm{C} 1$ and $\mathrm{C} 2$ are connected using Delaunay triangulation. Let $\mathrm{T}$ be a Delaunay triangle included inside the contour $\mathrm{C} 1$. The circumcenter $\mathrm{V}$ of $\mathrm{T}$ is located close to the inner skeleton of the 2-D shape described by $\mathrm{C} 1$ (V is the dual Voronoi vertex of $\mathrm{T}$ ). Let $\mathrm{P}$ be the closest point to $\mathrm{V}$ in the adjacent cross-section $\mathrm{C} 2$. The tetraedron generated by $\mathrm{T}$ and $\mathrm{P}$ is included inside the volume delimited by the surface to be reconstructed. More generally, we track 


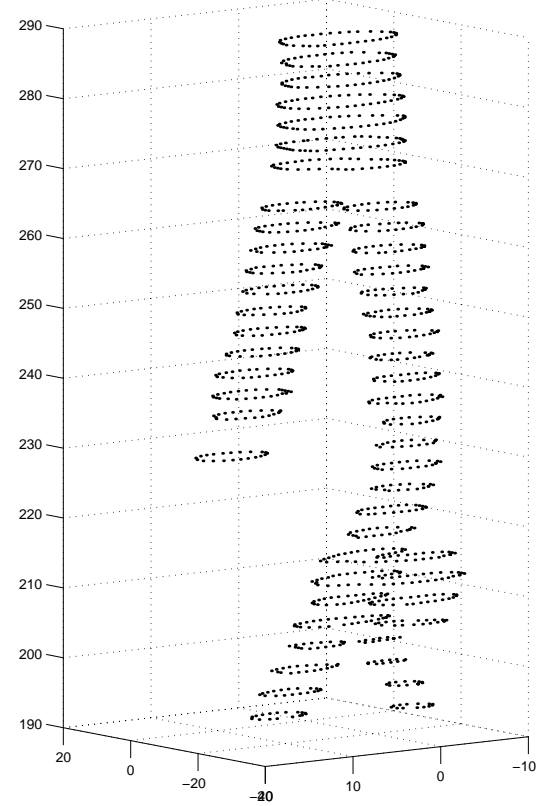

FiguRE 3. Slice contours obtained from the scanner images.

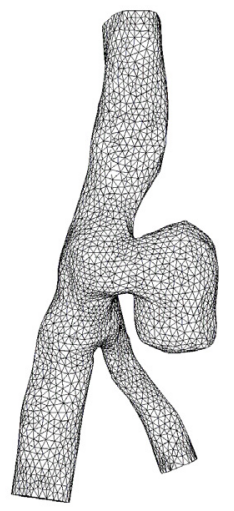

Figure 4. Surface mesh obtained from the slice contours by Toulouse - The second bifurcation.

inner tetraedra included in the object, by overlaying Voronoi diagrams of the two adjacent cross-sections. An inner tetrahedron is built from each inner Voronoi vertex but also from each intersection between inner Voronoi edges. An important property for subsequent computation was to ensure the convexity of the volume delimited by two consecutive sections when the latters are convex, Nuage ensuring this property.

Model III. First, the data points are fitted to a cubic spline, then equally re-sampled on it and finally noise is reduced on the new data points. Then, two successive slices are projected in a same plane and a 2D constrained Delaunay's triangulation is built. The surface triangles are then extracted by elevation of the two planes. Result is presented on Figure 5. Compared to the Model II, bifurcations are rougher but branches are smoother and less twisted. 

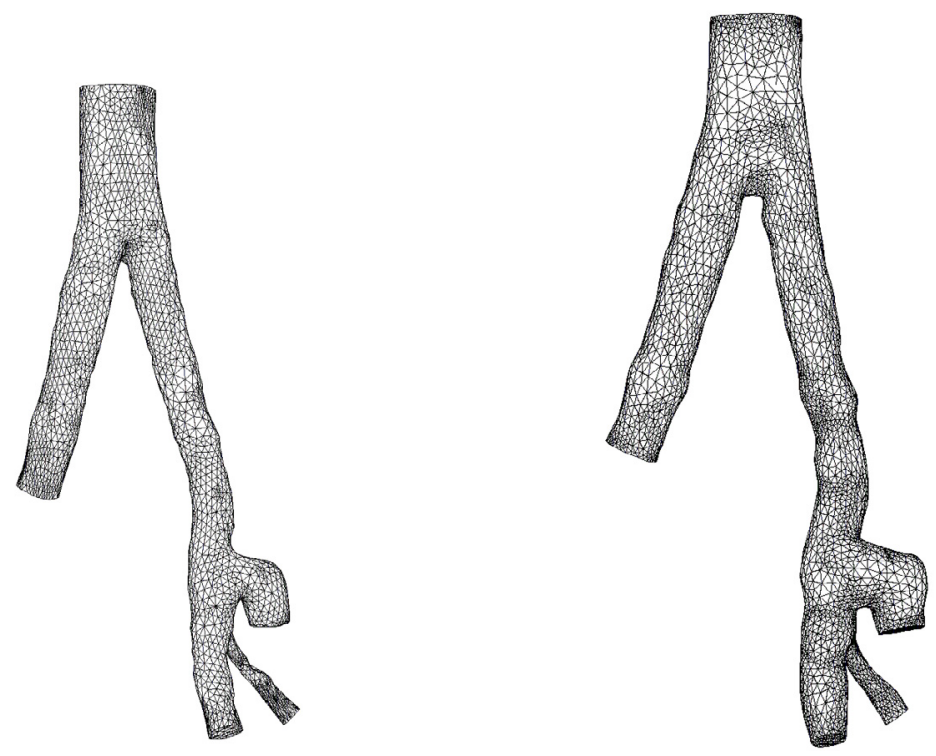

Figure 5. Surface mesh obtained from the slice contours Model II (left) and Model III (right).

\section{From FACETing to COMPUtational MEShes}

The triangulations obtained from the images need further treatment before computations. The operation, which leads to a computational mesh from a faceted surface, is composed of three main steps. Step 1 builds a computation-oriented surface triangulation. This triangulation is transformed into a geometrical model by step 2. Finally, the computational mesh is obtained in step 3.

The step 1 deals mainly with ends of the fluid domain. Indeed, nothing ensures that sections are crosssections. So, the edges of the inlet and outlet sections are labeled. A mesh scan of the facets gives the triangles with a labeled edge, which belong to the vessel wall and not to the vessel section. Once these facets are known, their normals are computed. The local axis, orthogonal to all the normals, is then determined by a least-square method. Thus, sections are corrected to be orthogonal to this axis. In order to avoid flow disturbance induced by boundary condition close to the exploration volume and to take into account the upstream and downstream effects on the flow of the complex vessel geometry (combination of curved vessels, possible branching segment, wall lesions and slight taper), straight segments in the direction of the local axis computed are added. The new inlet and outlet cross sections are labeled for boundary condition assignation. Moreover, with a cross section and a straight duct at the domain entry, the fluid is conveyed through an entry length from the entrance cross section, at which a Dirichlet condition is assigned via time-dependent uniform injection velocity. Furthermore, with cross sections and straight ducts at the exits, the pressure can be assumed to be uniform at the domain exit, at which Neumann no-stress conditions are prescribed. Available tools allow now to perform this stage automatically.

In the case of the congenital cerebral aneurism, the region of interest defined from the image set is too big. A smaller exploration volume is thus selected by cutting the region of interest with arbitrary planes. The resulting exploration volume is composed of a 3D curved trunk and its diseased bifurcation. This operation step needs 
human intervention. The resulting inlet and outlet sections are neither cross sections nor discretized. In the following step, the edges of the inlet and outlet sections are labeled and these sections discretized. Step 1 is then applied.

Step 2 corresponds to surface remeshing using GAMMA tools YAMS [2], in order to smooth the surface and to obtain a geometrical model. Note that this geometrical model keeps the main local curvatures of the input domain.

Step 3 provides a finite-element triangulation (e.g. [1]). From this surface triangulation the tetrahedron mesh is provided by the fast INRIA's software GHS3D [4].

\section{Numerical FLUID SIMUlations}

We compare two simulation results obtained from model II and III of the iliac aneurism (Fig. 6). Results ensures the robustness of the code used. Note that the similar results observed in both models are not totally surprising, as steps 2 and 3 are the same in both cases. However, the input meshes are quite different : Model II still needs more hand operations before the automatic step 1 than Model III.
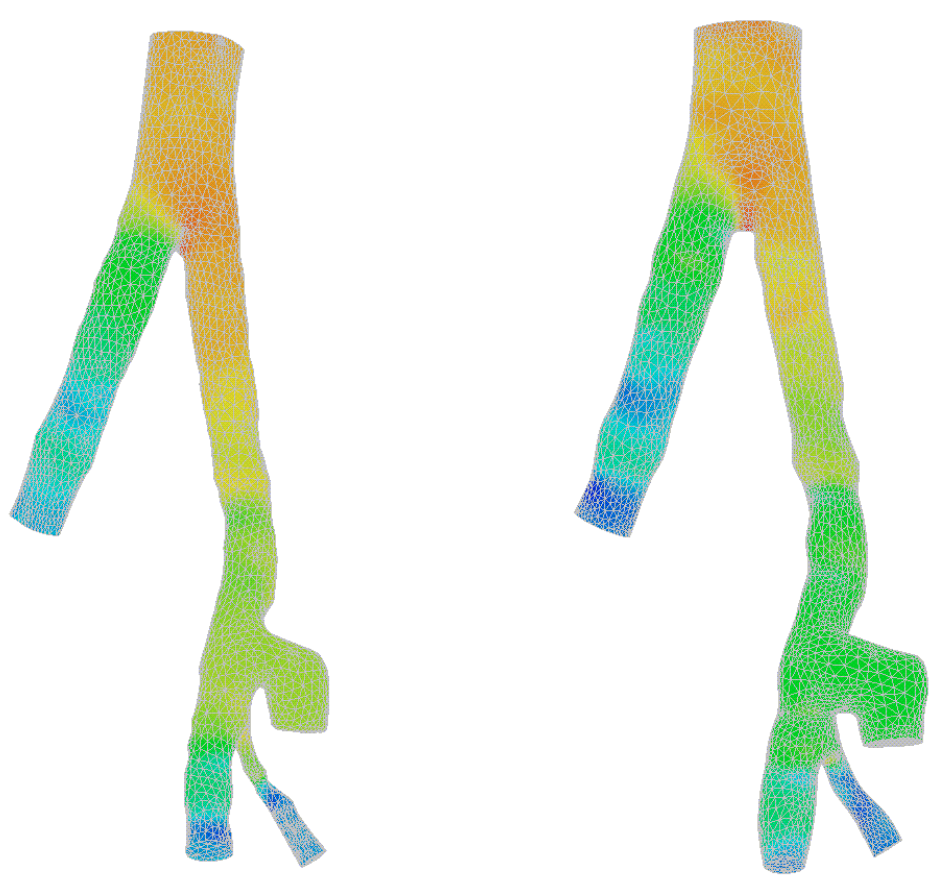

Figure 6. Pressure field in the second bifurcation of the iliac aneurism in Model II (left) and Model III (right) - Re $=750$. 


\section{Conclusion}

The faceting issued from iso-surface processing of the medical images gives faster geometrical models but needs a complete data set, thus a good image acquisition. In case of poor-quality images, or in case of partially saved slices of the scan set as for the iliac aneurism, the contour method is proved to be useful.

\section{REFERENCES}

[1] P.G. Ciarlet, The finite element method for elliptic problems, Studies in Mathematics and its applications, Vol. 4, NorthHolland, 1978.

[2] P. Frey, A fully automatic adaptive isotropic surface remeshing procedure, INRIA's Research Report 0252, 2001.

[3] K. Krissian, G. Malandain, N. Ayache, Model based multiscale detection and reconstruction of 3D vessels, INRIA's Research Report 3442, 1998.

[4] P.L. George, F. Hecht, E.Saltel, TetMesh distributed by SIMULOG, http://www.simulog.fr/tetmesh/.

[5] J-B. Mossa, Simulation d'une bifurcation artérielle, Report CERFACS, 2001.

[6] E. Saltel, article in preparation, 2002.

[7] T.K.F. Da, L'interpolation de formes, Phd thesis, Nice University, 2002. 